\title{
New Year and coronavirus
}

\author{
Chang-Ju Kim (Di) https://orcid.org/0000-0003-4749-5795
}

The year 2020 has begun. Coronavirus, which started in China from the beginning of the New Year, struck the world. Although the world is committed to the prevention and treatment of coronavirus pneumonia, the number of deaths and infections in China is increasing day by day.

Coronaviruses are a group of large enveloped, positive-sense, single-stranded RNA viruses belonging to the order Nidovirales, family Coronaviridae, subfamily Coronavirinae. With the high prevalence of coronaviruses, wide distribution, large genetic diversity, frequent recombination of the genome and increased activity at the human interface, these viruses represent a constant threat to human health. This fact became evident at the end of 2019 and early 2020. The new coronavirus has been found in Wuhan, China, to rapidly spread the large scale of respiratory diseases, including pneumonia.

Exercise is known to boost immunity. When immunity drops, we are vulnerable to infectious diseases. When immunity increases, we are in a state of developing autoimmune diseases. Thus, increased immunity means that immunity is harmoniously balanced. China is the country where many exercises such as Tai Chi and Kung Fu originated, and it is a nation that does many of these exercises. It is ironic that the corona virus began and became popular in China. There are limits to potentiate immunity with exercise.
This infection changed our way of life. Most people wear masks and avoid crowds. The opening of the school was delayed, and training and academic conferences were postponed indefinitely. The economic activity of each country and individual is also very limited. Everything in the world seems to have stopped before this viral infectious disease.

In order to win the war against this infectious disease, we must pay attention to the first personal hygiene. Next, we need to limit the movement of people and refrain from meetings. Finally, it is necessary to develop therapeutics and vaccination drugs.

The progress of science is still too difficult to reach its destination, and the development of medicine seems too slow. It's been a while since spring, but spring still seems to be far away.

\section{CONFLICT OF INTEREST}

No potential conflict of interest relevant to this article was reported.

President of the Korean Society of Exercise Rehabilitation Department of Physiology, College of Medicine Kyung Hee University 26 Kyungheedae-ro, Dongdaemun-gu, Seoul 02447, Korea E-mail: changju@khu.ac.kr 